\title{
La différence entre ne pas faire et ne rien faire
}

\author{
Christoph Bosshard \\ Dr méd., vice-président de la FMH, responsable du département Données, démographie et qualité
}

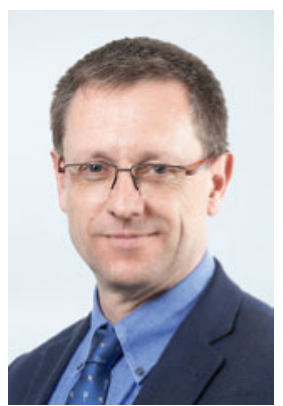

Il y a 20 ans environ, dans le cadre d'une bourse postdoctorale, j'ai eu l'opportunité de participer aux consultations d'un professeur ordinaire en chirurgie de la main. Cela a été pour moi l'occasion de voir comment il gérait non seulement les situations médicales complexes mais aussi les patients impatients. Un jour, alors qu'il venait de prescrire une IRM, je me suis permis de lui demander pourquoi il avait pris cette décision, qui ne me semblait pas indiquée pour le diagnostic. Il m'a répondu en souriant: "Aucune indication médicale ne justifie cette IRM mais le rendez-vous est dans six à huit semaines et sans lui, le patient n'aurait pas été disposé à poursuivre d'ici là sur la voie thérapeutique qui lui fera du bien.» Cette situation me revient souvent à l'esprit. Je ne sais malheureusement pas si, comme soupçonné, l'IRM avait été superflue. Mais j'ai appris qu'il fallait investir beaucoup plus de temps et de travail de persuasion pour accompagner et aider nos patients à supporter et accepter une situation, que pour agir ou faire. Sans compter que le temps disponible pour leur expliquer et les soutenir diminue partout un peu plus. Dans ce contexte, les éventuelles raisons de sombrer dans une forme d'hyperactivité sont toutes trouvées: personne ne veut laisser quelque chose au hasard, qui plus tard pourrait lui être reproché. On veut avoir tout essayé! Le temps, c'est de l'argent et l'absentéisme au travail est aussi un problème de la médecine. Aujourd'hui, les patients ne restent que quelques jours à l'hôpital alors qu'avant, avec le même diagnostic, ils y auraient passé plusieurs semaines. Cette concentration laisse peu de place au temps. Si auparavant, il fallait que je justifie à mes mentors pourquoi avoir prescrit une mesure diagnostique plutôt qu'une autre, j'entends aujourd'hui que, malgré les DRG, nos jeunes confrères en formation doivent rendre des comptes s'ils ne sont PAS intervenus. Cette peur de l'omission fait aussi partie de notre culture de la méfiance de plus en plus marquée. A cause d'elle toujours plus de ressources sont allouées à l'administration, à la documentation et aussi parfois à des mesures médicales qui, en définitive, ne servent qu'à étayer cette documentation et non le diagnostic. Pourtant la loi exige partout des critères EAE, ou justement peut-être pas partout? Dans le cadre des assurances sociales, lorsque nous discutons de nou- velles techniques ou que nous les comparons aux méthodes actuelles, nous parlons aussi d'efficacité, d'adéquation et d'économicité. Afin d'établir un aperçu des fondements scientifiques en lien avec Choosing Wisely ou Smarter Medicine et de susciter le débat, la FMH publie dans le présent numéro un document de base et la prise de position du Comité central de la FMH avec des propositions claires à ce sujet. Par ce biais, il souligne que le corps médical veut aussi empoigner ce défi, comme

\section{Il faut beaucoup plus de travail de persuasion pour accompagner et aider nos patients à supporter et accepter une situation que pour agir ou faire.}

il l'a déjà fait sur les questions de surconsommation [1], de guidelines [2] et de décision partagée [3]. Au-delà des bases scientifiques claires, ne-pas-faire exige aussi un travail de persuasion auprès du corps médical, l'acceptation des patients et de toute la société qui ne cesse d'exiger des «actes». Nous ne réussirons que si ce processus, mené par les médecins dans une relation médecin-patient basée sur la confiance et allégée des travaux administratifs, arrive à trouver sa place entre les espaces libres nécessaires pour que, dans certains cas justifiés, les médecins puissent bénéficier de marge de manœuvre par rapport aux listes Choosing Wisely ou aux guidelines. En effet, tous deux sont principalement axés sur la morbidité et ne tiennent pas suffisamment compte de la complexité de nos patients de plus en plus souvent polymorbides. En règle générale, ces recommandations devraient nous aider à investir pleinement notre réflexion médicale dans les cas complexes, et à faire valoir notre expérience et nos connaissances du cas particulier bien au-delà de la médecine et de l'évidence scientifique.

\section{Références}

1 Comité central de la FMH. Position de la FMH. Réduire la surconsommation, augmenter la qualité des soins. Bull méd suisses. 2016;97(7):244-5.

2 FMH/DDQ. Position de la FMH. Recommandations pour la pratique clinique (RPC): conditions et application. Bull méd suisses. 2014;95(3):52-3.

3 FMH/DDQ. Position de la FMH. Décision partagée. Bull méd suisses. 2014;95(50):1890-1. 